\title{
Is there long-term value of pathology scoring in immunoglobu- lin A nephropathy? A validation study of the Oxford Classification for IgA Nephropathy (VALIGA) update
}

Rosanna Coppo ${ }^{1}$, Graziella D’Arrigo ${ }^{2}$, Giovanni Tripepi ${ }^{2}$, Maria Luisa Russo ${ }^{1}$, Ian S.D Roberts ${ }^{3}$, Shubha Bellur ${ }^{3}$, Daniel Cattran ${ }^{4}$, Terence H. Cook ${ }^{5}$, John Feehally ${ }^{6}$, Vladimir Tesar ${ }^{7}$, Dita Maixnerova ${ }^{7}$, Licia Peruzzi ${ }^{8}$, Alessandro Amore ${ }^{8}$, Sigrid Lundberg ${ }^{9}$, Anna Maria Di Palma ${ }^{10}$, Loreto Gesualdo ${ }^{10}$, Francesco Emma ${ }^{11}$, Cristiana Rollino ${ }^{12}$, Manuel Praga ${ }^{13}$, Luigi Biancone ${ }^{14}$, Antonello Pani ${ }^{15}$, Sandro Feriozzi ${ }^{16}$, Rosaria Polci ${ }^{16}$, Jonathan Barratt ${ }^{6}$, Lucia Del Vecchio $^{17}$, Francesco Locatelli ${ }^{17}$, Alessandro Pierucci ${ }^{18}$, Yasar Caliskan ${ }^{19}$, Agnieszka Perkowska-Ptasinska ${ }^{20}$, Magdalena Durlik ${ }^{20}$, Elisabetta Moggia $^{21}$, José C. Ballarin ${ }^{22}$, Jack F.M. Wetzels ${ }^{23}$, Dimitris Goumenos ${ }^{24}$, Marios Papasotiriou ${ }^{24}$, Kresimir Galesic ${ }^{25}$, Luka Toric ${ }^{25}$, Aikaterini Papagianni ${ }^{26}$, Maria Stangou ${ }^{26}$, Luisa Benozzi ${ }^{27}$, Stefano Cusinato $^{27}$, Ulla Berg ${ }^{28}$, Rezan Topaloglu ${ }^{29}$, Milena Maggio ${ }^{30}$, Mai Ots-Rosenberg ${ }^{31}$, Marco D’Amico $^{32}$, Colin Geddes ${ }^{33}$, Olga Balafa ${ }^{34}$, Marco Quaglia ${ }^{35}$, Raffaella Cravero ${ }^{36}$, Calogero Lino Cirami ${ }^{37}$, Bengt Fellstrom ${ }^{38}$, Jürgen Floege ${ }^{39}$, Jesus Egido ${ }^{40}$, Francesca Mallamaci $^{2}$ and Carmine Zoccali ${ }^{2}$; for the ERA-EDTA Immunonephrology Working Group

${ }^{1}$ Fondazione Ricerca Molinette, Turin, Piemonte, Italy, ${ }^{2}$ CNR-IFC, Epidemiology, Reggio Calabria, Italy, ${ }^{3}$ Cellular Pathology, Oxford University Hospital, Oxford, UK, ${ }^{4}$ University, Toronto GH, Toronto, ON, Canada, ${ }^{5}$ Department of Nephrology, Imperial College, London, UK, ${ }^{6}$ Department of Nephrology, Leicester General Hospital, Leicester, UK, ${ }^{7}$ Nephrology, General University Hospital, Prague, Czech Republic, ${ }^{8}$ Nephrology, Regina Margherita Hospital, Turin, Italy, ${ }^{9}$ Department of Nephrology, Karolinska Institutet, Stockholm, Sweden, ${ }^{10}$ Department of Nephrology, BFU, Bari, Italy, ${ }^{11}$ Department of Nephrology, Bambino Gesù Children's Hospital - IRCCS, Rome, Italy, ${ }^{12}$ Department of Nephrology and Dialysis, OSGB, Turin, Italy, ${ }^{13}$ Department of Nephrology, H12Octubre, Madrid, Spain, ${ }^{14}$ Department of Nephrology, CSST, Turin, Italy, ${ }^{15}$ Department of Nephrology, AOGB, Cagliari, Italy, ${ }^{16}$ Department of Nephrology, Belcolle Hospital, Viterbo, Italy, ${ }^{17}$ Department of Nephrology, OAM, Lecco, Italy, ${ }^{18}$ Department of Nephrology, Sapienza University, Rome, Italy, ${ }^{19}$ Nephrology, Istanbul University, Istanbul, Turkey, ${ }^{20}$ Department of Transplantation Medicine and Nephrology, Medical University of Warsaw, Warsaw, Poland, ${ }^{21}$ Department of Nephrology, Santa Croce Hospital, Cuneo, Italy, ${ }^{22}$ Department of Nephrology, Puigvert, Barcelona, Spain, ${ }^{23}$ Department of Nephrology and Pathology, Radboud University Medical Center, Nijmegen, The Netherlands, ${ }^{24}$ Department of Nephrology and Kidney Transplantation, University Hospital of Patras, Patras, Greece, ${ }^{25}$ Department of Nephrology, Dubrava University, Zagreb, Croatia, ${ }^{26}$ Department of Nephrology, Aristotle University of Thessaloniki, Thessaloniki, Greece, ${ }^{27}$ Department of Nephrology, Borgomanero, Italy, ${ }^{28}$ Division of Pediatrics, Department of Clinical Science, Intervention and Technology, Huddinge, Sweden, ${ }^{29}$ Department of Pediatric Nephrology, Hacettepe University Faculty of Medicine Ankara, Turkey, ${ }^{30}$ Department of Nephrology, Hospital Maggiore di Lodi, Lodi, Italy, ${ }^{31}$ Department of Nephrology, Tartu University Clinics, Tartu, Estonia, ${ }^{32}$ Nephrology, S. Anna Hospital, Como, Colorado, Italy, ${ }^{33}$ Glasgow Renal and Transplant Unit, Queen Elizabeth University Hospital, Glasgow, UK, ${ }^{34}$ Department of Nephrology, Medical School University of Ioannina, Ioannina, Greece, ${ }^{35}$ Department of Nephrology, Maggiore della Carità Hospital, Piem, Onte Orientale University, Novara, Italy, ${ }^{36}$ Nephrology, Degli Infermi Hospital, Biella, Italy, ${ }^{37}$ Department of Nephrology, Careggi Hospital, Florence, Italy, ${ }^{38}$ Renal Department, University of Uppsala, Uppsala, Sweden, ${ }^{39}$ Division of Nephrology, Rheinisch-Westfälische Technische Hochschule Aachen, Aachen, Germany and ${ }^{40}$ Department of Nephrology, Fundacion Jimenez Diaz, CIBERDEM, Madrid, Spain

Correspondence and offprint requests to: Rosanna Coppo; E-mail: rosanna.coppo@unito.it; Twitter handle: @carminezoccali

\section{ABSTRACT}

Background. It is unknown whether renal pathology lesions in immunoglobulin A nephropathy (IgAN) correlate with renal outcomes over decades of follow-up.
Methods. In 1130 patients of the original Validation Study of the Oxford Classification for IgA Nephropathy (VALIGA) cohort, we studied the relationship between the MEST score (mesangial hypercellularity, M; endocapillary hypercellularity, 
E; segmental glomerulosclerosis, S; tubular atrophy/interstitial fibrosis, T), crescents (C) and other histological lesions with both a combined renal endpoint [50\% estimated glomerular filtration rate (eGFR) loss or kidney failure] and the rate of eGFR decline over a follow-up period extending to 35 years [median 7 years (interquartile range 4.1-10.8)].

Results. In this extended analysis, M1, S1 and T1-T2 lesions as well as the whole MEST score were independently related with the combined endpoint $(\mathrm{P}<0.01)$, and there was no effect modification by age for these associations, suggesting that they may be valid in children and in adults as well. Only $\mathrm{T}$ lesions were associated with the rate of eGFR loss in the whole cohort, whereas $\mathrm{C}$ showed this association only in patients not treated with immunosuppression. In separate prognostic analyses, the whole set of pathology lesions provided a gain in discrimination power over the clinical variables alone, which was similar at 5 years $(+2.0 \%)$ and for the whole follow-up $(+1.8 \%)$. A similar benefit was observed for risk reclassification analyses $(+2.7 \%$ and $+2.4 \%)$.

Conclusion. Long-term follow-up analyses of the VALIGA cohort showed that the independent relationship between kidney biopsy findings and the risk of progression towards kidney failure in IgAN remains unchanged across all age groups and decades after the renal biopsy.

Keywords: IgA nephropathy, progression, renal biopsy, risk factors

\section{INTRODUCTION}

Immunoglobulin A nephropathy (IgAN) is a common glomerular disease, accounting for 22\% of renal biopsies in Europe and up to $39.5 \%$ in Asia $[1,2]$. Apart from extremely mild or severely aggressive forms, in most cases, IgAN has a slow relentless progression with loss of renal function and kidney failure in 10$30 \%$ of cases over 10 years [3]. As in other renal diseases, severe proteinuria and hypertension at renal biopsy and during followup are strongly correlated with the risk of progression to kidney failure in IgAN [4]. The inherent biological variation of these two biomarkers requires time-averaged estimates of proteinuria and blood pressure (BP) over a minimum of 2 years to ensure a sufficiently accurate prognostication of renal outcomes in this disease [5]. The value of renal biopsy features to predict progression of IgAN was established by the Oxford Classification for IgA Nephropathy, a study which demonstrated that mesangial hypercellularity $(M)$, endocapillary hypercellularity (E), segmental glomerulosclerosis (S) and tubular atrophy/interstitial fibrosis (T) (forming the MEST score) were directly related with incident renal outcomes independent of clinical data [estimated glomerular filtration rate (eGFR), proteinuria, mean arterial pressure (MAP)] at the time of renal biopsy and during followup (time-averaged proteinuria and time-averaged MAP values) [6-8]. A recent large collaborative study demonstrated the additional value of crescents (C) for outcome prognostication, indicating the value of adopting a MEST-C score for full evaluation of renal pathology risk factors in $\operatorname{IgAN}[9,10]$.

A number of studies have confirmed the relationship of the MEST score with renal outcomes [11]. The Validation Study of the Oxford Classification for IgA Nephropathy (VALIGA) was one of the largest of these studies and consisted of 1147 cases of IgAN from 13 European countries [12]. It validated the prognostic value of MST lesions across the whole spectrum of clinical features of this disease and retrospectively assessed the effects of corticosteroid treatment in patients with selected MEST scores [13]. A subsequent collaborative study that pooled data from 901 patients in the VALIGA, Oxford and North America validation studies showed that combining the original MEST score with clinical data at the time of renal biopsy improved prediction of the risk for the renal endpoint of eGFR decline $>50 \%$ or kidney failure as compared with baseline clinical data only [14]. Furthermore, this combined approach predicted the renal outcome with accuracy similar to the purely clinical approach based on longitudinal data collection over 2 years. Thus, combining baseline clinical data and histology findings allows immediate prediction of the evolution of IgAN at the time of biopsy, obviating the need for 2 years of data collection demanded by the clinical score alone $[5,14]$.

The importance of being able to determine the risk for severe chronic kidney disease (CKD) and kidney failure (G5 defined by Kidney Disease Improving Global Outcome) over extended follow-up periods, including lifetime risk, has been emphasized in population-based modelling studies [15-17]. The issue is of relevance for a disease such as IgAN because, due to likely differences in pathophysiological pathways, this disease has a variable progression rate. Some patients may not develop kidney failure during the first year of follow-up but may still have a substantial risk for this outcome over decades. Thus, investigating the relationship of the MEST-C score with renal outcomes over prolonged follow-up is an issue of clinical relevance.

With this in mind, the present study was conceived to test the relationship of the MEST-C score at diagnosis with renal outcomes in the VALIGA cohort over an extended follow-up period.

\section{MATERIALS AND METHODS}

\section{Patients}

VALIGA enrolled 1147 European patients with primary IgAN from 53 centres in 13 European countries [12]. Patients with Henoch-Schönlein nephritis, chronic hepatitis, diabetes or cancer were excluded. Each renal biopsy was centrally rescored by the pathology review centre in Oxford, UK. The last VALIGA record was in September 2011. For the purpose of the present analysis, follow-up was updated until December 2015.

\section{Clinical data and definitions}

The definition of children, measurement of proteinuria and MAP in children and in adults and time-averaged proteinuria and time-averaged MAP have been described in the VALIGA study [12]. Estimated glomerular filtration rate (eGFR) was estimated using the four-variable Modification of Diet and Renal Disease (MDRD) formula in adults, to be consistent with previous reports $[7,12]$, and the Schwartz formula in children (constant $K=0.55$ ), with a maximum eGFR set at $120 \mathrm{~mL} / \mathrm{min} /$ $1.73 \mathrm{~m}^{2}$, as previously described $[8,17]$. Kidney failure was 
defined as an eGFR $\leq 15 \mathrm{~mL} / \mathrm{min} / 1.73 \mathrm{~m}^{2}$. Corticosteroid/immunosuppressive treatment was evaluated on an intent-to-treat basis, regardless of the type or duration of therapy.

\section{Pathology review}

Renal biopsies were scored according to the Oxford classification: M0/M1 ( $\leq 50 \%$ of glomeruli with $<4$ mesangial cells/ mesangial area/ $>50 \%$ of glomeruli with $\geq 4$ mesangial cells/mesangial area), E0/E1 (absent/present), S0/S1 (absent/present) and T0/T1/T2 $(<25,25-50,>50 \%)$. In addition, arteriosclerosis and extracapillary proliferation with cellular or fibrocellular $\mathrm{C}$ were categorized as absent/present according to the Oxford definition. Moreover, $\mathrm{C}$ was subdivided into two classes of glomeruli with C1 $>$ and C2 $\geq 25 \%$ of glomeruli [9].

\section{Outcomes}

Two clinical outcomes were considered to evaluate the predictive value of renal pathology variables (consistent with the Oxford study and VALIGA first report), progression to the combined endpoint of a 50\% reduction in eGFR or kidney failure and the rate of renal function decline (eGFR slope) during follow-up.

\section{Statistical methods}

Normally distributed data were expressed as mean \pm standard deviation, non-normally distributed variables as median and interquartile range (IQR) and binary data as a percentage, as appropriate. The follow-up period was summarized as median and range.

The renal function loss over time was assessed on an individual basis by fitting a straight line of the relationship 'eGFR versus time', and the slope was adopted as an index of eGFR decline, with the latter expressed as $\mathrm{mL} / \mathrm{min} / 1.73 \mathrm{~m}^{2} /$ year. Multivariable country-weighted linear regression models were used to identify the independent correlates of eGFR slope. In these analyses we included M, E, S, T and also C and arteriosclerosis (which were significant at univariate analysis) and adjusted for age, gender, baseline eGFR, baseline body mass index (BMI), baseline MAP and baseline proteinuria. Additional multivariable sensitivity analyses including time-averaged values of MAP and proteinuria were calculated as previously described [7]. Data were expressed as the standardized regression coefficient $(\beta)$ and $P$-value.

Time-to-event analysis was performed by country-stratified Cox regression analysis and the independent association of isolated and combined components of MEST, $\mathrm{C}$ and atherosclerosis was assessed in Cox models, including key demographic (age and gender) and clinical variables [mean arterial pressure and proteinuria (either baseline or time-averaged) and BMI]. Results were expressed as the hazard ratios (HRs), 95\% confidence intervals (CIs) and P-values.

The discriminant power of individual components of the MEST score, $\mathrm{C}$ and arteriosclerosis (up to 5 and 35 years) to predict the combined endpoint was assessed by Harrell's $C$ test [18] and the corresponding reclassification ability by the Integrated Discrimination Improvement (IDI) [19]. For all tests, P-values $<0.05$ were considered statistically significant.
Table 1. Baseline characteristics at renal biopsy of 1130 patients with IgAN

\begin{tabular}{|c|c|}
\hline Age (years) & $35 \pm 16$ \\
\hline Paediatrics subjects ( $<18$ years of age), $\%$ & 15 \\
\hline Ethnicity (Caucasian/African/Asian/other), \% & $97.6 / 0.6 / 0.6 / 1.2$ \\
\hline eGFR $\left(\mathrm{mL} / \mathrm{min} / 1.73 \mathrm{~m}^{2}\right)$ & $74 \pm 31$ \\
\hline \multicolumn{2}{|l|}{ CKD stage, $\%$} \\
\hline 1 or 2 & 63.7 \\
\hline 3 & 28.5 \\
\hline 4 & 7.8 \\
\hline Male, \% & 73 \\
\hline $\operatorname{MAP}(\mathrm{mmHg})^{\mathrm{a}}$ & $98 \pm 13$ \\
\hline $\mathrm{SBP}(\mathrm{mmHg})^{\mathrm{a}}$ & $131 \pm 20$ \\
\hline $\mathrm{DBP}(\mathrm{mmHg})^{\mathrm{a}}$ & $81 \pm 12$ \\
\hline Proteinuria (g/day) ${ }^{\mathrm{a}}$, median (IQR) & $1.2(0.5-2.4)$ \\
\hline \multicolumn{2}{|l|}{ Proteinuria ( $\%$ of patients) } \\
\hline$<0.5 \mathrm{~g} /$ day & 21 \\
\hline$\geq 0.5-1 \mathrm{~g} /$ day & 20 \\
\hline$\geq 1-3 \mathrm{~g} /$ day & 40 \\
\hline$\geq 3$ g/day & 19 \\
\hline \multicolumn{2}{|l|}{ Pathology features, $\%$} \\
\hline M1 & 28 \\
\hline E1 & 11 \\
\hline S1 & 70 \\
\hline $\mathrm{T} 1-2$ & 21 \\
\hline $\mathrm{C} 1-2, \%$ & 10.5 \\
\hline Arteriosclerosis (presence), \% & 27 \\
\hline
\end{tabular}

$\overline{{ }^{a} \text { In subjects }<18 \text { years of age, blood pressure values were corrected as previously detailed }}$ $[8,17]$ and proteinuria was reported per $1.73 \mathrm{~m}^{2}$ body surface area. Pathology findings are defined according to Oxford classification [6]: $\mathrm{C} 1$ in $<25 \%$ and $\mathrm{C} 2$ in $\geq 25 \%$ of glomeruli. Data presented as mean \pm standard deviation unless stated otherwise. SBP: systolic blood pressure; DBP: diastolic blood pressure.

All calculations were performed using SPSS for Windows, version 22 (IBM, Armonk, NY, USA) and STATA for Windows, version 13 (StataCorp, College Station, TX, USA).

\section{RESULTS}

\section{VALIGA follow-up cohort}

Centres contributing to VALIGA were asked to update the clinical records of 1147 patients from 55 centres of the original VALIGA study (see list in Appendix 1). After the exclusion of 17 patients with eGFR $<15 \mathrm{~mL} / \mathrm{min} / 1.73 \mathrm{~m}^{2}$ at renal biopsy (the kidney failure threshold, i.e. one of the study endpoints; see 'Materials and methods' section), the follow-up analysis was performed in 1130 patients. In the present analysis, the median follow-up was 7 years (IQR 4.1-10.8) and ranged from 0.08 to 35 years, prolonged by $51 \%$ from the median follow-up of 4.7 years (IQR 2.4-7.9) of the original VALIGA study.

Demographic, clinical and pathology data at renal biopsy are reported in Table 1 and those during follow-up (time-averaged data, treatments and renal outcomes) in Table 2. All patients were residents of Europe and $97.6 \%$ were Caucasian. Most patients had their renal biopsy performed when in CKD Stages 1-3. Twenty-eight percent of renal biopsies showed M1, 11\% $\mathrm{E} 1,70 \% \mathrm{~S} 1$ and $21 \% \mathrm{~T} 1$ or T2. T2 lesions were infrequent (3.6\%), hence $\mathrm{T} 1$ and $\mathrm{T} 2$ were combined for statistical analysis. Overall, C was present in $10.5 \%$ of cases, $8.6 \%$ had $<25 \%$ of glomeruli involved (C1) and only $1.9 \%$ had $\mathrm{C}$ in $\geq 25 \%$ of glomeruli (C2). Hence $\mathrm{C} 1$ and $\mathrm{C} 2$ were combined for statistical 
Table 2. Follow-up data and clinical outcomes

\begin{tabular}{|c|c|}
\hline Duration of follow-up (years), median (IQR) & $7.0(0.08-35.0)$ \\
\hline MAP (mmHg, time averaged $)^{\mathrm{a}}$ & $95 \pm 9$ \\
\hline SBP $(\mathrm{mmHg} \text {, time averaged })^{\mathrm{a}}$ & $128 \pm 13$ \\
\hline DBP (mmHg, time averaged $)^{a}$ & $79 \pm 8$ \\
\hline $\begin{array}{l}\text { Number of antihypertensive drugs, median } \\
\text { (IQR) }\end{array}$ & $1(1.0-2.0)$ \\
\hline $\begin{array}{l}\text { Patients on } 1,2 \text { or }>2 \text { antihypertensive drugs, } \\
\%\end{array}$ & $36,22,18$ \\
\hline $\begin{array}{l}\text { Proteinuria (g/day, time averaged })^{\mathrm{a}} \text {, median } \\
\text { (IQR) }\end{array}$ & $0.8(0.4-1.6)$ \\
\hline \multicolumn{2}{|l|}{ Median time-averaged proteinuria $(\mathrm{g} / \text { day })^{\mathrm{a}}, \%$} \\
\hline$<0.5$ & 32 \\
\hline$\geq 0.5-1$ & 26 \\
\hline$\geq 1-1.5$ & 15 \\
\hline$\geq 1.5-2$ & 10 \\
\hline$\geq 2$ & 17 \\
\hline \multicolumn{2}{|l|}{ Treatments, \% } \\
\hline Corticosteroid/immunosuppressive drugs & 48 \\
\hline $\begin{array}{l}\text { ACE inhibitors and/or angiotensin II } \\
\text { blockers }\end{array}$ & 87 \\
\hline Fish oil & 15 \\
\hline Tonsillectomy & 3 \\
\hline \multicolumn{2}{|l|}{ Clinical outcome data } \\
\hline $\begin{array}{l}\text { Rate of decline in renal function }(\mathrm{mL} / \mathrm{min} / \\
\left.1.73 \mathrm{~m}^{2} / \text { year }\right)\end{array}$ & $-1.8 \pm 7.2$ \\
\hline $50 \%$ decrease in eGFR, $n(\%)$ & $255(23.5)$ \\
\hline $\operatorname{ESRD}\left(<15 \mathrm{~mL} / \mathrm{min} / 1.73 \mathrm{~m}^{2}\right), n(\%)$ & $212(18.8)$ \\
\hline $50 \%$ decrease in eGFR or ESRD, $n(\%)$ & $279(24.7)$ \\
\hline
\end{tabular}

analysis. Twenty-seven percent had arteriosclerosis. During the follow-up, $87 \%$ of patients were treated with angiotensin converting enzyme (ACE) inhibitors or angiotensin II blockers and $48 \%$ with corticosteroids and/or other immunosuppressive drugs.

\section{Correlations between renal pathology lesions and outcomes}

Over the long-term follow-up of the VALIGA cohort, 212 patients (18.8\%) developed incident kidney failure (CKD Stage G5) and 255 (23.5\%) had a 50\% decline in eGFR. Overall, 279 (24.7\%) reached the combined endpoint [50\% decline in eGFR or kidney failure over the follow-up period (Supplementary data, Figure S1)]. The adjusted ratio HR for the combined endpoint associated with a $1 \mathrm{U}$ increase in the MEST score was 1.62 (95\% CI 1.40-1.87; P < 0.001). In multivariable survival analyses over long-term follow-up (Table 3), clinical variables (i.e. baseline eGFR, proteinuria and MAP) at renal biopsy were robustly associated with this outcome measure. In the whole cohort, M1, S1 and T1-T2 were independently related with the combined endpoint (Table 3). There was no effect modification by age for these associations (P for interaction ranged from 0.09 to 0.90 ), suggesting that they may be valid in children and in adults as well. Further multivariable analyses including time-averaged values of MAP and proteinuria rather than the corresponding baseline values confirmed these associations (data not shown).
In the multivariate analysis of the whole study population over an extended follow-up period, eGFR, MAP and proteinuria at the time of renal biopsy were significantly associated with the rate of renal function loss (Table 4). The MEST score was associated with prediction of the rate of loss of GFR ( $\beta$-coefficient $-0.16, \mathrm{P}<0.001)$. Among components of the MEST score, only $\mathrm{T}$ was significantly related to the same outcome variable $(\mathrm{P}<0.001)$. There was no effect modification by age on this association $(\mathrm{P}$ for interaction $=0.77)$. In the subpopulation that never received corticosteroids or other immunosuppressive drugs (Table 4, right column), T, baseline eGFR and baseline proteinuria maintained an independent relationship with eGFR loss over time. In the same non-treated subpopulation, C emerged as a significant correlate of the outcome variable $(\mathrm{P}=0.01) \quad$ (Table 4 , right column). In the small group of patients with $\mathrm{C} 2$ lesions, no independent relationship with the combined renal endpoint over and above the mere presence of $\mathrm{C}$ was detectable.

Additional multivariable analyses including time-averaged values of MAP and proteinuria over the follow-up period rather than the corresponding baseline values substantially confirmed these associations (data not shown).

\section{Prognostic value of pathology features over long follow- up times}

The adjusted HR of the MEST score for the combined endpoint at the median follow-up value of 7 years (patients at risk, $n=557)$ was $1.65(95 \% \mathrm{CI} 1.38-1.96 ; \mathrm{P}<0.001)$ and the $\mathrm{HR}$ at 25 years was 1.62 (95\% CI $1.40-1.87 ; \mathrm{P}<0.001)$.

To estimate the prognostic value of the individual components of the MEST score, $\mathrm{C}$ and arteriosclerosis, we performed a discriminant analysis using the Harrell's $\mathrm{C}$ index. We calculated the discriminatory power of the simple risk model based on clinical variables (age, gender, baseline eGFR, baseline BMI, time-averaged MAP and time-averaged proteinuria) for a follow-up period of $\leq 5$ years [median 4.0 years (range 0.08 5.0)] and for the whole observation period that extended to 35 years [median 7 years (range $0.08-35$ )]. Over the extended observation period, the discriminatory power of the basic model remained virtually the same as the 5 -year model (83.7 versus 85.2\%). Individual components of the MEST score, the sum of the MEST score, $\mathrm{C}$ and arteriosclerosis added a predictive power to the basic clinical model in the 35-year follow-up period of $0-0.8 \%$ very similar to that over the 5 -year follow-up (from $0 \%$ to $0.5 \%$ ). The simultaneous inclusion of all pathology features (MEST, C and arteriosclerosis) to the basic model provided a gain in discrimination that did not change over the 5- and 35-year follow-up period (2.0 and 1.8\%, respectively).

In the risk reclassification analyses by IDI, only T1-T2 significantly improved risk reclassification in the 5-year (IDI $+2.3 \%, \mathrm{P}=0.003$ ) and prolonged follow-up cohorts (IDI $+1.5 \%, \mathrm{P}<0.001$ ). S1 provided a significant improvement in risk reclassification only in the prolonged follow-up cohort $(+0.5 \%, \mathrm{P}=0.048)$. The improvement in risk reclassification provided by the remaining pathology features ranged from $-0.05 \%$ to $0.30 \%$ at 5 years ( $\mathrm{P}$ ranged from 0.37 to 0.68 ) and 
Table 3. Multivariable Cox regression analysis for the risk of reaching the combined renal endpoint of $50 \%$ decline in eGFR or kidney failure

\begin{tabular}{lcc} 
Characteristics & All patients $(n=1130)$ & $\begin{array}{c}\text { Patients never treated with } \\
\text { corticosteroid/immunosuppressors } \\
\text { during the follow-up }(n=582)\end{array}$ \\
M1 & $1.34(1.02-1.75), \mathrm{P}=0.037^{*}$ & $1.83(1.23-2.75), \mathrm{P}=0.003^{*}$ \\
E1 & $1.17(0.79-1.74), \mathrm{P}=0.43$ & $0.83(0.45-1.53), \mathrm{P}=0.55$ \\
S1 & $1.61(1.10-2.36), \mathrm{P}=0.01^{*}$ & $1.54(0.89-2.66), \mathrm{P}=0.13$ \\
T1-2 & $2.46(1.80-3.36), \mathrm{P}<0.001^{*}$ & $2.73(1.66-4.47), \mathrm{P}<0.001^{*}$ \\
Crescents (C1-2) & $0.85(0.55-1.30), \mathrm{P}=0.44$ & $1.81(0.90-3.64), \mathrm{P}=0.10$ \\
Arteriosclerosis & $1.19(0.89-1.58), \mathrm{P}=0.24$ & $1.20(0.79-1.82), \mathrm{P}=0.39$ \\
Age & $1.00(0.99-1.01), \mathrm{P}=0.60$ & $1.00(0.99-1.02), \mathrm{P}=0.61$ \\
Gender (male) & $0.90(0.67-1.22), \mathrm{P}=0.51$ & $1.02(0.64-1.63), \mathrm{P}=0.93$ \\
Baseline eGFR & $0.99(0.98-0.99), \mathrm{P}<0.001^{*}$ & $0.98(0.97-0.99), \mathrm{P}=0.001^{*}$ \\
Baseline MAP & $1.01(1.00-1.02), \mathrm{P}=0.005^{*}$ & $1.01(1.00-1.03), \mathrm{P}=0.03^{*}$ \\
Baseline proteinuria & $1.19(1.12-1.26), \mathrm{P}<0.001^{*}$ & $1.21(1.11-1.32), \mathrm{P}<0.001^{*}$ \\
Baseline BMI & $1.01(0.98-1.05), \mathrm{P}=0.46$ & $1.02(0.97-1.06), \mathrm{P}=0.50$ \\
\hline
\end{tabular}

Dependent variable: 50\% decrease in eGFR or ESRD. Data are presented as HR, 95\% CI and P-value. ${ }^{*}$ Statistically significant P-values. Pathology findings are defined according to the Oxford classification [6]: M, E, S, T (T1 and T2 pooled), crescents (C1 and C2 pooled) and arteriosclerosis (significant at univariate analysis) adjusted for age, gender and baseline data of eGFR, MAP, proteinuria and BMI. The analysis is country weighted.

Table 4. Multivariable linear regression analysis of the rate of renal function decline (eGFR slope)

\begin{tabular}{lcc} 
Characteristics & $\begin{array}{c}\text { All patients } \\
(n=1130)\end{array}$ & $\begin{array}{c}\text { Patients never treated with } \\
\text { corticosteroid/immunosuppressors } \\
\text { during the follow-up }(n=582)\end{array}$ \\
\hline M1 & $-0.03(\mathrm{P}=0.28)$ & $-0.06(\mathrm{P}=0.18)$ \\
E1 & $-0.06(\mathrm{P}=0.07)$ & $0.08(\mathrm{P}=0.07)$ \\
S1 & $-0.05(\mathrm{P}=0.14)$ & $-0.07(\mathrm{P}=0.14)$ \\
T1-2 & $-0.16\left(\mathrm{P}<0.001^{*}\right)$ & $-0.14\left(\mathrm{P}=0.003^{*}\right)$ \\
Crescents $(\mathrm{C} 1-2)$ & $0.002(\mathrm{P}=0.95)$ & $-0.11\left(\mathrm{P}=0.01^{\star}\right)$ \\
Arteriosclerosis & $-0.002(\mathrm{P}=0.94)$ & $0.03(\mathrm{P}=0.51)$ \\
Gender (male $)$ & $0.02(\mathrm{P}=0.42)$ & $-0.006(\mathrm{P}=0.89)$ \\
Age & $-0.01(\mathrm{P}=0.76)$ & $0.004(\mathrm{P}=0.94)$ \\
Baseline eGFR & $-0.18\left(\mathrm{P}<0.001^{\star}\right)$ & $-0.11\left(\mathrm{P}=0.05^{\star}\right)$ \\
Baseline MAP & $-0.12\left(\mathrm{P}<0.001^{*}\right)$ & $-0.08(\mathrm{P}=0.10)$ \\
Baseline proteinuria $-0.08\left(\mathrm{P}=0.02^{*}\right)$ & $-0.13\left(\mathrm{P}=0.004^{\star}\right)$ \\
Baseline BMI & $0.001(\mathrm{P}=0.97)$ & $-0.02(\mathrm{P}=0.74)$ \\
\hline
\end{tabular}

Dependent variable: rate of renal function decline. Data are presented as standardized regression coefficient (beta) and P-value. ${ }^{*}$ Statistically significant P-values. Pathology findings are defined according to the Oxford classification [6]: M, E, S, T, crescents and arteriosclerosis adjusted for age, gender and baseline data of eGFR, MAP and proteinuria. The analysis is country weighted.

from 0.05 to $0.16 \%$ at 35 years ( $P$ ranged from 0.22 to 0.66 ). The IDI using the whole set of pathology lesions (MEST, $\mathrm{C}$ and arteriosclerosis) was $2.7 \%$ at 5 years $(\mathrm{P}=0.001)$ and $2.4 \%$ at 35 years $(\mathrm{P}<0.001)$.

\section{DISCUSSION}

The VALIGA analysis of pooled European cohorts of patients with IgAN validated the prediction value of the Oxford MEST score for the combined renal endpoint of 50\% eGFR loss or kidney failure or eGFR decline that was independent of baseline eGFR, proteinuria and MAP over a median follow-up of about 4 years [12]. The independent relationship of the MEST score and $\mathrm{C}$ with these same outcomes over prolonged follow-up has not been addressed until now.
As in other glomerular diseases, the renal lesions in IgAN undergo dynamic changes due to evolution of the underlying pathological process and/or the effect of treatments. Some lesions, for instance $\mathrm{E}$ and $\mathrm{C}$, are particularly subject to change, as demonstrated by repeat renal biopsy studies that show their decrease or disappearance over time after corticosteroids, cyclophosphamide and mycophenolate mofetil treatment or their progression to glomerular sclerosis [20-22]. Regression of M has similarly been detected in a repeated renal biopsy study after treatment with corticosteroid and immunosuppressive treatment in children with IgAN [23]. Also, podocyte lesions detected in a subset of patients with the S1 score were reduced after 6 months of corticosteroid treatment [22]. In contrast, progression of glomerular sclerosis and tubular interstitial damage is also found in repeated renal biopsy studies and is likely to represent unfavourable evolution of these active lesions [20,22].

The prognostic value of these potentially modifiable pathological features detected at renal biopsy over a prolonged period of follow-up is unknown. Even though repeated renal biopsies could provide important information to guide clinical decisions during the disease course, clinicians rarely undertake a second biopsy, even in the setting of persistent proteinuria despite optimal supportive care. Therefore, knowing whether the information provided by the first diagnostic renal biopsy remains valid in the long term is a relevant clinical question in IgAN. To investigate this, we extended the follow-up of the IgAN VALIGA cohort of $>1000$ patients [12], all with centrally scored MESTC.

This long-term country-weighted analysis of the VALIGA cohort, which includes adults and children, shows that the MEST score remains directly associated with the combined renal endpoint over observation periods extending to $>3$ decades. In addition, in patients who never received corticosteroid/immunosuppressive treatment, the presence of $\mathrm{C}$ was related to the rate of renal function decline, independent of the MEST score and other risk factors. The lack of effect on the combined endpoint (which is a severe outcome for the patient, with $50 \%$ 
loss of eGFR or kidney failure) is probably due to the fact that the patients enrolled in the VALIGA study had a modest median annual eGFR loss $(1.8 \pm 7.2 \mathrm{~mL} / \mathrm{min} /$ year $)$ and were scarcely crescentic with a low percentage of $\mathrm{C} 2$ lesions. Consistent with many studies $[11,24,25]$, including the original VALIGA [12], T was the pathological lesion most robustly associated with adverse renal outcomes during extended follow-up, a finding that was age and immunosuppression independent, confirming previous explorative studies indicating that chronic changes may be highly predictive even in early biopsies [26]. The percentage of T2 lesions was so low that T1 and T2 were pooled for statistical analysis, hence no independent value of T1 or T2 could be assessed. Therapies targeted at pathways promoting early tubular/interstitial damage are essential to limit the progression of kidney disease, and this is supported by the findings of this extended VALIGA study [27]. The M1 lesions also had a significant impact on the outcome decades after the renal biopsy particularly in patients who did not receive immunosuppression. Hence the search for effective and safe therapies acting on modifiable histological risk factors, including mesangial cells activation, is crucial to the goal of limiting the transformation of active glomerular lesions to irreversible fibrotic scars.

The improvement in prognostication by adding pathology features to baseline clinical data in patients with IgAN was reported for the first time in a Japanese study using their national histological classification $[28,29]$. They showed an improvement in prediction of $5 \%$, similar to the $6 \%$ improvement in the $\mathrm{C}$ index over $>5$ years reported using the MEST score in a multicentre study analysing pooled multicontinental cohorts [14]. The improvement in risk discrimination and reclassification of the whole series of histological lesions considered in the present analysis was 1.8 and $2.4 \%$, respectively. These small improvements in the present long-term follow-up cohort likely reflect the already high discriminatory value of the model with clinical data only [14]. Although this might be considered a limited contribution to the risk factors driving the natural history of IgAN, it is of a similar magnitude to other appreciated risk factors, for example, a seven-single-nucleotide polymorphism genetic risk score, which explained $4.7 \%$ of overall IgAN risk according to large genome-wide association studies [30].

This long-term follow-up analysis strengthens the observations of the first VALIGA study and subsequent large collaborative studies, indicating that the MEST-C lesions remain associated with renal outcomes over a very long time window, independent of the treatment administered. These findings suggest that in IgAN, alongside clinical risk factors, the severity of renal damage at diagnosis dictates the risk of progression towards kidney failure.

Clinical trials do not often consider baseline renal biopsy features. Baseline biopsy data may offer material for predictive analytics beyond the simple presence of persistent proteinuria that has generally been used as enrolment criterion, enriching the clinical trial population with active and potentially progressive patients. In the era of precision nephrology, pathology lesions, particularly mesangial proliferation, podocytopathy and crescents, as well as the extent of tubular and interstitial damage, may represent useful criteria for selecting patients for clinical trials at high risk for adverse renal outcomes.

In conclusion, the long-term follow-up of the VALIGA cohort shows that the independent relationship of the MEST-C score found on kidney biopsy remains associated with the risk of progression towards kidney failure in patients with IgAN decades after the renal biopsy. These results further highlight the potential value of key histological alterations for designing future clinical trials in IgAN.

\section{SUPPLEMENTARY DATA}

Supplementary data are available at ndt online.

\section{ACKNOWLEDGEMENTS}

The VALIGA study was granted by the first research call of the European Renal Association-European Dialysis and Transplant Association in 2009. The authors are deeply grateful to Stéphan Troyanov for his great work on the first analysis of the original VALIGA database.

\section{FUNDING}

The study was supported by a research grant of the European Renal Association-European Dialysis and Transplant Association.

\section{CONFLICT OF INTEREST STATEMENT}

The article has been seen and approved by all the authors and it is not under consideration for publication elsewhere in a similar form in any language.

\section{REFERENCES}

1. Magistroni R, D'Agati VD, Appel GB et al. New developments in the genetics, pathogenesis, and therapy of IgA nephropathy. Kidney Int 2015; 88: 974-989

2. O'Shaughnessy M, Hogan S, Bawana D et al. Glomerular disease frequencies by race, sex, and region: from the International Kidney Biopsy Survey. Nephrol Dial Transpl 2018; 33: 661-669

3. Barbour S, Reich H. An update on predicting renal progression in IgA nephropathy. Curr Opin Nephrol Hypertens 2018; 27: 214-220

4. Coppo R. Clinical and histological risk factors for progression of IgA nephropathy: an update in children, young and adult patients. J Nephrol 2017; 30: 339-346

5. Bartosik LP, Lajoie G, Sugar L et al. Predicting progression in IgA nephropathy. Am J Kidney Dis 2001; 38: 728-735

6. Roberts IS, Cook HT, Troyanov S et al. The Oxford classification of IgA nephropathy: pathology definitions, correlations, and reproducibility. Kidney Int 2009; 76: 546-556

7. Cattran DC, Coppo R, Cook HT et al. The Oxford classification of IgA nephropathy: rationale, clinicopathological correlations, and classification. Kidney Int 2009; 76: 534-545

8. Coppo R, Troyanov S, Camilla R et al. The Oxford IgA nephropathy clinicopathological classification is valid for children as well as adults. Kidney Int 2010; 77: 921-927

9. Haas M, Verhave JC, Liu ZH et al. A Multicenter study of the predictive value of crescents in IgA nephropathy. J Am Soc Nephrol 2017; 28: 691-701

10. Trimarchi H, Barratt J, Cattran DC et al. Oxford classification of IgA nephropathy 2016: an update from the IgA Nephropathy Classification Working Group. Kidney Int 2017; 91: 1014-1021 
11. Roberts IS. Oxford classification of immunoglobulin A nephropathy: an update. Curr Opin Nephrol Hypertens 2013; 22: 281-286

12. Coppo R, Troyanov S, Bellur S et al. Validation of the Oxford classification of IgA nephropathy in cohorts with different presentations and treatments. Kidney Int 2014; 86: 828-836

13. Tesar V, Troyanov S, Bellur S et al. Corticosteroids in IgA nephropathy: a retrospective analysis from the VALIGA study. J Am Soc Nephrol 2015; 26: 2248-2258

14. Barbour SJ, Espino-Hernandez G, Reich HN et al. The MEST score provides earlier risk prediction in IgA nephropathy. Kidney Int 2016; 89: 167-175

15. Grams ME, Chow EK, Segev DL et al. Lifetime incidence of CKD stages 3-5 in the United States. Am J Kidney Dis 2013; 62: 245-252

16. Levey AS, Bosch JP, Lewis JB et al. A more accurate method to estimate glomerular filtration rate from serum creatinine: a new prediction equation. Modification of Diet in Renal Disease Study Group. Ann Intern Med 1999; 130: 461-470

17. Coppo R, Lofaro D, Camilla RR et al. Risk factors for progression in children and young adults with IgA nephropathy: an analysis of 261 cases from the VALIGA European cohort. Pediatr Nephrol 2017; 32: 139-150

18. Harrell FE Jr, Lee KL, Mark DB. Multivariable prognostic models: issues in developing models, evaluating assumptions and adequacy, and measuring and reducing errors. Stat Med 1996; 15: 361-387

19. Pencina MJ, D'Agostino RB Sr, D'Agostino RB Jr et al. Evaluating the added predictive ability of a new marker: from area under the ROC curve to reclassification and beyond. Stat Med 2008; 27: 157-172

20. Shen XH, Liang SS, Chen HM et al. Reversal of active glomerular lesions after immunosuppressive therapy in patients with IgA nephropathy: a repeatbiopsy based observation. J Nephrol 2015; 28: 441-449

21. Beckwith H, Medjeral-Thomas N, Galliford J et al. Mycophenolate mofetil therapy in immunoglobulin A nephropathy: histological changes after treatment. Nephrol Dial Transplant 2017; 32: i123-i128

22. Hou JH, Le WB, Chen $\mathrm{N}$ et al. Mycophenolate mofetil combined with prednisone versus full-dose prednisone in IgA Nephropathy with active proliferative lesions: a randomized controlled trial. Am J Kidney Dis 2017; 69: 788-795

23. Yoshikawa N, Honda M, Iijima K et al. Steroid treatment for severe childhood IgA nephropathy: a randomized, controlled trial. Clin J Am Soc Nephrol 2006; 1: 511-517

24. Kaihan $\mathrm{AB}$, Yasuda $\mathrm{Y}$, Katsuno $\mathrm{T}$ et al. The Japanese Histologic Classification and T-score in the Oxford classification system could predict renal outcome in Japanese IgA nephropathy patients. Clin Exp Nephrol 2017; 21: 986-994

25. Zhu X, Li H, Liu Y et al. Tubular atrophy/interstitial fibrosis scores of Oxford classification combined with proteinuria level at biopsy provides earlier risk prediction in lgA nephropathy. Sci Rep 2017; 7: 1100

26. Lemley KV, Lafayette RA, Derby G et al. Prediction of early progression in recently diagnosed IgA nephropathy. Nephrol Dial Transplant 2008; 23: 213-222

27. Hodgkins KS, Schnaper HW. Tubulointerstitial injury and the progression of chronic kidney disease. Pediatr Nephrol 2012; 27: 901-909

28. Tanaka S, Ninomiya T, Katafuchi $R$ et al. Development and validation of a prediction rule using the Oxford classification in IgA nephropathy. Clin J Am Soc Nephrol 2013; 8: 2082-2090

29. Kawamura $\mathrm{T}$, Joh $\mathrm{K}$, Okonogi $\mathrm{H}$ et al. A histologic classification of IgA nephropathy for predicting long-term prognosis: emphasis on end-stage renal disease. J Nephrol 2013; 26: 350-357

30. Kiryluk K, Li Y, Sanna-Cherchi S et al. Geographic differences in genetic susceptibility to IgA nephropathy: GWAS replication study and geospatial risk analysis. PLoS Genet 2012; 8: e1002765

Received: 28.6.2018; Editorial decision: 13.8.2018

\section{APPENDIX 1}

The VALIGA centres' list of nephrologists includes the following (asterisks mark the centres that sent the update by 2016):

V. Tesar, D. Maixnerova (Nephrology, First Faculty of Medicine and General University Hospital, Prague, Czech Republic)*; S. Lundberg (Nephrology, Karolinska University
Hospital, KarolinskaInstitutet, Stockholm, Sweden)*; L. Gesualdo (Nephrology, Emergency and Organ Transplantation, University of Bari "Aldo Moro", Foggia-Bari, Italy)*; F. Emma, L. Fuiano (Nephrology, Pediatrico Bambino Gesù Hospital, Rome, Italy)*; G. Beltrame, C. Rollino (Nephrology, San Giovanni Bosco Hospital, Turin, Italy)*; R. Coppo, A. Amore, R. Camilla, L. Peruzzi (Nephrology, Regina Margherita Children's Hospital, Turin, Italy)*; M. Praga (Nephrology, Hospital 12 de Octubre, Madrid, Spain)*; S. Feriozzi, R. Polci, (Nephrology, Belcolle Hospital, Viterbo, Italy)*; G. Segoloni, L. Colla (Nephrology, S. Giovanni Battista University Hospital, Turin, Italy)*; A. Pani, A. Angioi, L. Piras (Nephrology, G. Brotzu Hospital, Cagliari, Italy)*; J. Feehally (John Walls Renal Unit, Leicester General Hospital, Leicester, United Kingdom)* ${ }^{*}$ G. Cancarini, S. Ravera (Nephrology, Spedali Civili University Hospital, Brescia, Italy); M. Durlik (Transplantation Medicine and Nephrology, Warsaw Medical University, Warsaw, Poland)*; E. Moggia (Nephrology, Santa Croce Hospital, Cuneo, Italy)*; J. Ballarin (Nephrology, Fundacion Puigvert, Barcelona, Spain)*; S. Di Giulio (Nephrology, San Camillo Forlanini Hospital, Rome, Italy); F. Pugliese, I. Serriello (Nephrology, Policlinico Umberto I University Hospital, Rome, Italy)*; Y. Caliskan, M. Sever, I. Kilicaslan (Nephrology, Internal Medicine, Istanbul Faculty of Medicine, Istanbul, Turkey)*; F. Locatelli, L. Del Vecchio (Nephrology, A. Manzoni Hospital, Lecco, Italy)** J.F.M. Wetzels, H. Peters (Nephrology and Pathology, Radboud University Nijmegen Medical Center, Nijmegen, The Netherlands)*; U. Berg (Pediatrics, Department of Clinical Science, Intervention and Technology, Huddinge, Sweden)*; F. Carvalho, A.C. da Costa Ferreira (Nephrology, Hospital de Curry Cabral, Lisbon, Portugal)*; M. Maggio (Nephrology, Hospital Maggiore di Lodi, Lodi, Italy)*; A. Wiecek (Nephrology, Endocrinology and Metabolic Diseases, Silesian University of Medicine, Katowice, Poland); M. Ots-Rosenberg (Nephrology, Tartu University Clinics, Tartu, Estonia)*; R. Magistroni (Nephrology, Policlinic of Modena and Reggio Emilia; Modena, Italy); R. Topaloglu, Y. Bilginer (Pediatric Nephrology and Rheumatology, Hacettepe University, Ankara, Turkey)** M. D'Amico (Nephrology, S. Anna Hospital, Como, Italy)*; M. Stangou (Nephrology, Hippokration General Hospital, Aristotle University of Thessaloniki, Thessaloniki, Greece)*; F. Giacchino (Nephrology, Ivrea Hospital, Ivrea, Italy)*; D. Goumenos, M. Papastirou, (Nephrology, University Hospital of Patras, Patras, Greece)*; K. Galesic, L. Toric (Nephrology, University Hospital Dubrava, Zagreb, Croatia)*; C. Geddes (Renal Unit, Western Infirmary Glasgow, Glasgow, UK)*; K. Siamopoulos, O. Balafa (Nephrology, Medical School University of Ioanina, Ioannina, Greece $)^{*} ;$ M. Galliani (Nephrology, S. Pertini Hospital, Rome, Italy); P. Stratta, M. Quaglia (Nephrology, Maggiore della Carità Hospital, Piemonte Orientale University, Novara, Italy)*; R. Bergia, R. Cravero (Nephrology, Degli Infermi Hospital, Biella, Italy)*; M. Salvadori, L. Cirami (Nephrology, Careggi Hospital, Florence, Italy)** B. Fellstrom, H. Kloster Smerud (Renal Department, University of Uppsala, Uppsala, Sweden)*; 
F. Ferrario, T. Stellato (Nephropathology, San Gerardo Hospital, Monza, Italy); J. Egido, C. Martin (Nephrology, Fundacion Jimenez Diaz, Madrid, Spain) ${ }^{\star}$; J. Floege, F. Eitner, T. Rauen (Nephrology and Immunology, Medizinische Klinik II, University of Aachen, Aachen, Germany)*; A. Lupo, P. Bernich (Nephrology, University of Verona, Verona, Italy); P. Menè (Nephrology, S. Andrea Hospital, Rome, Italy); M. Morosetti (Nephrology, Grassi Hospital, Ostia, Italy); C. van Kooten, T. Rabelink, M.E.J. Reinders (Nephrology, Leiden University Medical Centre, Leiden, The Netherlands)*; J.M. Boria Grinyo (Nephrology, Hospital Bellvitge, Barcelona, Spain); S. Cusinato, L. Benozzi (Nephrology, Borgomanero Hospital, Borgomanero, Italy)*; S. Savoldi, C. Licata (Nephrology, Civile Hospital, Ciriè, Italy)*; M. MizerskaWasiak, M. Roszkowska-Blaim (Pediatrics, Medical University of Warsaw, Warsaw, Poland); G. Martina, A. Messuerotti (Nephrology, Chivasso Hospital, Chivasso, Italy) ${ }^{*}$; A. Dal Canton, C. Esposito, C. Migotto (Nephrology Units, S. Matteo Hospital and Maugeri Foundation, Pavia, Italy); G. Triolo, F. Mariano (Nephrology CTO, Turin, Italy)*; C. Pozzi (Nephrology, Bassini Hospital, Cinisello Balsamo, Italy) ${ }^{*}$; R. Boero (Nephrology, Martini Hospital, Turin, Italy)* .
The VALIGA centres' list of pathologists includes the following: G. Mazzucco (Turin, Italy); C. Giannakakis (Rome, Italy); E. Honsova (Prague, Czech Republic); B. Sundelin (Stockholm, Sweden); A.M. Di Palma (Foggia-Bari, Italy); F. Ferrario (Monza, Italy); E. Gutiérrez (Madrid, Spain); A.M. Asunis (Cagliari, Italy); J. Barratt (Leicester, United Kingdom); R. Tardanico (Brescia, Italy); A. PerkowskaPtasinska (Warsaw, Poland); J. Arce Terroba (Barcelona, Spain); M. Fortunato (Cuneo, Italy); A. Pantzaki (Thessaloniki, Greece); Y. Ozluk (Istanbul, Turkey); E. Steenbergen (Nijmegen, The Netherlands); M. Soderberg (Huddinge, Sweden); Z. Riispere (Tartu, Estonia); L. Furci (Modena, Italy); D. Orhan (Ankara, Turkey); D. Kipgen (Glasgow, UK); D. Casartelli (Lecco, Italy); D. GalesicLjubanovic (Zagreb, Croatia); H Gakiopoulou (Athens, Greece), E. Bertoni (Florence, Italy); P. Cannata Ortiz (Madrid, Spain); H. Karkoszka(Katowice, Poland), H.J. Groene (Heidelberg, Germany); A. Stoppacciaro (Rome, Italy); I. Bajema, J. Bruijn (Leiden, The Netherlands); X. Fulladosa Oliveras (Barcelona, Spain); J. Maldyk (Warsaw, Poland) and E. Ioachim (Ioannina, Greece). 